\title{
Rapid HPLC/UV method for analysis of urinary and plasma/serum paracetamol concentrations
}

\author{
Preethi Soysa* and Saman Kolambage \\ Department of Biochemistry and Molecular Biology, Faculty of Medicine, University of Colombo, Kynsey Road. Colombo 8.
}

\begin{abstract}
Paracetamol is one of the most commonly used over-the-counter pain relievers and fever-reducers. However, overdosing of paracetamol causes liver damage which may lead to patients' death upon delayed treatment. To provide accurate diagnosis and fast treatment of paracetamol poisoning, rapid analysis of paracetamol in patient tissues is necessary. In this study a rapid and simple high performance liquid chromatographic method was developed to analyse plasma/serum and urinary paracetamol using $100 \mu \mathrm{L}$ of specimen. Paracetamol is chromatographically resolved, with an isocratic mobile phase followed by UV detection after protein precipitation. $\beta$-Hydroxyethyltheophylline was used as the internal standard. The elution of paracetamol and the internal standard was achieved within 8 minutes. The calibration curve was linear over $0.25-200 \mathrm{mg} / \mathrm{L}$. This method produced excellent accuracy and precision in all matrices tested. Intra and inter day variability was less than $5 \%$. The limits of detection were $0.13 \mathrm{mg} / \mathrm{L}$ for plasma and $0.43 \mathrm{mg} / \mathrm{L}$ for urine, whereas the limits of quantification were $0.68 \mathrm{mg} / \mathrm{L}$ for plasma; and $2.25 \mathrm{mg} / \mathrm{L}$ for urine. No matrix effects were observed with endogenous substances. This method is applied to analyze paracetamol levels in serum of accidental or self poisioning patients.
\end{abstract}

Keywords: HPLC, paracetamol analysis, paracetamol overdose.

\section{INTRODUCTION}

Paracetamol, also known as acetaminophen, is one of the most commonly used over-the-counter analgesic and antipyretic drugs available ${ }^{1,2}$. Following oral administration and absorption from the gastrointestinal tract, paracetamol enters the blood, is distributed throughout the body and metabolised in the liver ${ }^{3}$. At therapeutic doses, paracetamol is largely converted to inactive metabolites by conjugation with sulphate or glucuronide and excreted within 24 hours. Paracetamol toxicity is likely to occur after a minimum ingestion of $140 \mathrm{mg} / \mathrm{kg}^{4}$. Hepatotoxicity may occur-7 when the enzymes responsible for catalysing sulphate and glucuronide conjugation become saturated, forcing metabolism to be increasingly dependent upon the cytochrome P450 system ${ }^{7,8}$. This results in formation of a toxic metabolite, $\mathrm{N}$-acetyl-p-benzo-quinone imine which is associated with hepatic damage in paracetamol overdosed patients 9 . At toxic doses liver damage may occur within 24 to 48 hours requiring fast reporting of patients' exposure levels, thus, rapid analysis of paracetamol in biological matrices is of great importance for clinical and forensic toxicologists.

Several high performance liquid chromatographic (HPLC) methods for analysis of paracetamol in urine ${ }^{10,11}$ and in serum ${ }^{12,13}$ are reported in literature. Several factors should be considered in developing an analytical method for detecting paracetamol levels. The sample volume is important specially with peadiatric patients. Analysis of any chemical in serum by HPLC requires removing of proteins from the test sample prior to analysis for the safety of the column and the instrument. Solvent extraction has been used in most methods ${ }^{13,14}$ for sample processing which removes serum proteins simultaneously. Extraction methods using organic solvents need lengthy sample processing time. Protein precipitation has been achieved in nonextraction methods by addition of acetonitrile ${ }^{15}$ for Glyburide analysis, perchloric acid ${ }^{16}$ for analysis of caffeine and paraxanthine, methano ${ }^{17}$ for the measurement of 10,11-dihydro-10-hydroxycarbamazepine and hydrochloric acid/ammonium sulphate ${ }^{18}$ in paracetamol analysis. Direct injection after filtering the sample through $0.45 \mu \mathrm{m}$ disposable filters ${ }^{19}$ was also reported in Dyphylline and Doxofylline in serum by HPLC. However, the use of filters measures only the protein unbound fraction of the drug present 
in serum. Addition of an organic solvent facilitates the dissociation of proteins from the drug by changing the polarity of the medium. In this study, deproteinisation was achieved by the addition of acetonitrile, enabling the total serum paracetamol to be quantified. Preparation of the internal standard in acetonitrile and addition of this mixture simultaneously achieved the presence of internal standard in the serum and the protein precipitation in sample processing. Adequate protein separation was achieved by centrifugation at high speed.

The method used earlier in our laboratory was a HPLC/UV method as described by Quattrone and Putnam with slight modifications in the running solvent ${ }^{13}$. In this method $\beta$-hydroxyethyltheophylline has been used as the internal standard and paracetamol was analysed after ethylacetate extraction. The running solvent used in the original method was acetonitrile:water $(1: 11)$ with acetic acid $(0.05 \%)$ and triethylamine $(0.08 \%)$, which was replaced by $10 \%$ acetonitrile for the extraction method used earlier in our laboratory ${ }^{13}$. Present method described in this paper eliminates the extraction of paracetamol with ethylacetate, which prolongs time for sample preparation. Our current method only involves addition of internal standard prepared in acetonitrile (100\%) to the test samples followed by centrifugation after a brief vortex mix. Processing of 20-24 samples were able to complete for the injection during the time that HPLC system is being equilibrated (less than $30 \mathrm{~min}$ ) with the mobile phase.

\section{METHODS AND MATERIALS}

HPLC grade acetonitrile was purchased from BDH (BDH Chemicals Ltd. Poole, England). $\beta$ hydroxyethyltheophylline was purchased from Sigma Chemicals, USA. Paracetamol ( $>99.99 \%$ purity) was gifted by Dr. MDJ Wijebandara and Mr. W Pathirana (Faculty of Medicine, University of Colombo). Purified de-ionized water was prepared using Milli Q50 water purification system (Millipore, USA).

Plasma samples used in this study to prepare standards were obtained from the Blood Bank of the National Hospital of Sri Lanka. The identities of the donors were not known. Fresh urine samples were used to prepare standards which were collected from healthy volunteers of both sexes, who have not taken paracetamol over three or more days.

As an application of the method, serum was analysed from the blood samples $(n=80)$ and sent to the laboratory for paracetamol analysis. Samples were brought to the laboratory by various sources including National
Hospital of Sri Lanka. In addition a forensic sample of one and half years old child who had the symptoms of paracetamol toxicity was analysed. This sample was obtained from the Department of Forensic Medicine and Toxicology, Faculty of Medicine, Colombo. The blood level of paracetamol in volunteers $(\mathrm{n}=5)$ were also analysed after obtaining ethical clearance from the ethical clearance committee (EC/08/132), Faculty of Medicine, Colombo.

HPLC was performed with Shimadzu LC 10AS solvent delivery system equipped with UV/VIS variable wavelength detector Shimadzu SPD 10A (Shimadzu Corporation, Japan), degasser Shimadzu DGU-14A (Shimadzu Corporation, Japan) and an integrator Shimadzu C-R8A (Shimadzu Corporation, Japan). Chromatographic resolution of paracetamol in serum and urine was achieved on a $3.9 \times 300 \mathrm{~mm}, \mu$ Bondpack C-18 HPLC column (Waters, Milford, MA) attached to a $10 \times 4.6 \mathrm{~mm}$ Spherisorb guard column (Waters, USA). Samples were injected with a syringe loading injector fitted with $20 \mu \mathrm{L}$ loop. Micro-centrifugation was performed using a BioFuge-Pico centrifuge (Heraeus Instruments, Germany).

Stock standard of paracetamol was made by accurately measuring $0.15 \mathrm{~g}$ of paracetamol in a $50 \mathrm{~mL}$ volumetric flask and dissolving it in a minimum volume of MilliQ water and diluted with the relevant matrix (plasma or urine) to make the stock standard to minimize matrix effects. The subsequent dilutions were prepared (0.25-200 $\left.\mathrm{mg} \mathrm{L}^{-1}\right)$ by diluting the stock standard with relevant matrix and used to construct the calibration curve. The matrix was initially screened to confirm the absence of paracetamol. The internal standard, used was $\beta$-hydroxyethyltheophylline at a concentration of $200 \mathrm{mg} \mathrm{L}^{-1}$ in acetonitrile (100\%). The chromatographic conditions were optimized to elute paracetamol without interfering the internal standard (at the specified concentration) and the substances present in matrix of serum/plasma or urine at a wavelength of $254 \mathrm{~nm}$. All standards were stored at $-20^{\circ} \mathrm{C}$.

Quality control (QC) materials were prepared from a base urine and plasma pool obtained from multiple anonymous donors at three concentration levels by spiking known amounts of paracetamol to achieve final concentrations of $12.5,25$ and $50 \mathrm{mg} \mathrm{L}^{-1}$ and 25,50 and $100 \mathrm{mg} \mathrm{L}^{-1}$ for plasma and urine respectively. Each QC material of plasma was then dispensed into $1.5 \mathrm{~mL}$ microcentrifuge tubes and stored at $-20^{\circ} \mathrm{C}$. QC samples were analyzed for method validation and during sample analysis to ensure the quality of the data. 
Human urine, or plasma sample was thawed, vortex mixed and aliquoted $(100 \mu \mathrm{L})$ into a microcentrifuge tube. The sample was spiked with the internal standard prepared in acetonitrile $(100 \mu \mathrm{L})$ and votex mixed. The proteins and other insoluble particulate were separated from plasma or urine by centrifuging at $10,000 \mathrm{~g}$ for $10 \mathrm{~min}$. The supernatant was then transferred to a clean micro centrifuge tube and recentrifuged at the same speed for additional $3 \mathrm{~min}$. The resultant plasma/serum supernatant $(25 \mu \mathrm{L})$ was injected on to the C18-HPLC column for chromatographic resolution and ultra violet (UV) detection. It was further diluted before analysis of urine samples by adding the mobile phase (1:1) for better resolution of the peaks. The mobile phase was prepared by mixing $120 \mathrm{~mL}$ of acetonitrile and $880 \mathrm{~mL}$ of water (12:88; acetonitrile: water). The flow rate was set at $1.0 \mathrm{~mL} \mathrm{~min}^{-1}$. Paracetamol and the internal standard were detected at the wave length of $254 \mathrm{~nm}$. Matrix was replaced with water for reagent blanks.

The present method was validated according to FDA guidelines ${ }^{20}$. The accuracy and intra and inter day precision was assessed by repeat measurement of the three QC materials in plasma or urine prepared similar to the unknown sample. The interferences due to co-eluting contaminants at the retention times of paracetamol or internal standard were also investigated to evaluate the specificity by analysing pre-screened plasma and urine blanks. With each set of test samples, blank plasma or urine with and without the internal standard were injected. Furthermore, since some foods and formulations of paracetamol contain caffeine, the potential interference from caffeine with the retention times of paracetamol and the internal standard was also tested. All the experiments were carried out at room temperature.

The calibration standards were prepared similar to unknown samples along with reagent blanks and QC samples. Peak area ratios of paracetamol standards to that of internal standard were plotted against the concentration to construct the calibration curve. The slope and the intercept of the calibration curves were calculated through least squares linear regression using MicrosoftExcel Program. The calibration curve was accepted if $\mathrm{R}^{2}$ $>0.99$. Sample sequence was constructed with a set of calibration standards, blanks, QCs and unknowns.

Slope and the intercept of the calibration curve were evaluated over 6 wks using 6 independent series of calibration standards prepared in urine and plasma matrix with paracetamol concentration ranging from 0.25 to $200 \mathrm{mg} \mathrm{L}^{-1}$. The linearity of the calibration curve was also investigated by repeat analysis of calibration standards prepared in the matrix for over $6 \mathrm{wks}$.
The robustness of the analytical method was evaluated by repeating the analysis under different experimental conditions; by changing the flow rate at $0.8 \mathrm{~mL} \mathrm{~min}^{-1}$ at a concentration of $12 \%$ acetonitrile and increasing the acetonitrile composition to $14 \%$ and running the solvent at $1.0 \mathrm{~mL} \mathrm{~min}^{-1}$.

Five lowest standard concentrations prepared in pre-screened blank plasma or urine were analyzed in replicates $(\mathrm{N}=6)$. The standard deviation (SD) obtained at each concentration was plotted against the concentration. The intercept at zero concentration $\left(\mathrm{SD}_{0}\right)$ obtained by extrapolation of the curve was used to evaluate the LOD and $\mathrm{LOQ}^{21}$. The LOD was calculated as the $3 * \mathrm{SD}_{0}$ and LOQ was $10 * \mathrm{SD}_{0}{ }^{21}$.

Known amounts of paracetamol were spiked into plasma $\left(12.5,25\right.$ and $\left.50 \mathrm{mg} \mathrm{L}^{-1}\right)$ and urine $(25,50$ and $\left.100 \mathrm{mg} \mathrm{L}^{-1}\right)$, at three concentration levels and the analysis was performed. Recovery was tested for paracetamol spiked into water at four concentrations covering the concentration ranges used for both plasma and urine $\left(10,25,50\right.$ and $\left.100 \mathrm{mg} \mathrm{L}^{-1}\right)$. The calibration curve constructed using standards with the same batch of spiked samples was used to calculate the paracetamol levels in each sample. The percentage recovery was determined using the calculated value to the true value.

The accuracy of the measurements was determined by evaluating the repeat measurements of plasma and urine QC samples $(n=6)$ prepared at three concentration levels; 12.5, 25 and $50 \mathrm{mg} \mathrm{L}^{-1}$ and 25, 50 and $100 \mathrm{mg} \mathrm{L}^{-1}$ for plasma and urine respectively. The inter day $(n=10)$ and intra day (for six successive weeks) precision of the analytical method was also investigated by repeat analysis of the same QC material and evaluating the coefficient of variation $(\% \mathrm{CV})$.

Long term stability of paracetamol was evaluated by repeat analysis of the $\mathrm{QC}$ material stored at $-20^{\circ} \mathrm{C}$ for over a period of six weeks ( $24 \mathrm{~h}, 1 \mathrm{wk}, 4 \mathrm{wks}$ and $6 \mathrm{wks})$. Post preparative stability was also investigated by measuring the levels of paracetamol by re-injecting previously prepared samples. The stability of the standards stored in the refrigerator at $4^{\circ} \mathrm{C}$ for $24 \mathrm{~h}$ to $6 \mathrm{~d}$ was also evaluated by repeat measurement of analytical standards. Freezethaw stability of plasma paracetamol was evaluated over 4 wks upto three freeze thaw cycles, which include after $24 \mathrm{~h}$, week 2 and week 4 using QC material stored at $-20^{\circ} \mathrm{C}$. 


\section{RESULTS}

The solvent composition and the flow rate were optimized to minimize matrix interferences. The mobile phase used in this assay provided well defined separation of paracetamol and the internal standard with excellent chromatographic resolution (Figure 1). The peaks were identified by the retention time and spiking of authentic standards to blank and test samples (plasma and urine). The paracetamol and the internal standard were eluted at $5.53 \pm 0.19$ minutes and $6.46 \pm 0.30$ minutes, respectively. No interfering co-eluting peaks were present either in plasma, serum or urine blanks due to endogenous substances or caffeine in formulations suggesting excellent selectivity of paracetamol (Figure 1). The calibration curve constructed with 10 working standards was linear over $0.25-200 \mathrm{mg} \mathrm{L}^{-1}$ for plasma and urine matrix with $\mathrm{R}^{2}$ typically exceeding 0.99 (Figure 2). The internal standard provided recovery correction improving the accuracy of the measurements. The excellent linearity of the calibration curve suggests adequacy of the $\beta$-hydroxyethyltheophylline as the internal standard for paracetamol analysis. The method robustness was excellent with acceptable variation in retention times (Table 1) after changing the percentage of organic content in the mobile phase and the flow rate with no co-eluting interferences. Despite changing the method parameters, the calibration curve produced linearity $\left(\mathrm{R}^{2}>0.99\right)$ suggesting the adaptability of the method (Table 1).

The gradient (mean \pm standard deviation) of the calibration curve made in plasma and urine remained stable over the whole concentration range, after 24 hours, one week, four weeks and six weeks $(0.0155 \pm 0.0003$ and $0.0167 \pm 0.0006$ for plasma and urine respectively) when stored at $-20^{\circ} \mathrm{C}$. The values were compatible with the slope from freshly made standards (Figure 2) suggesting short and long term stability of the paracetamol in the matrix with minimal intraday $(n=6)$ and interday $(n=10)$

Table 1: Study of chromatographic variables with plasma

\begin{tabular}{|c|c|c|c|}
\hline Chromatographic condition & $\begin{array}{l}\mathrm{RT}_{\mathrm{PCM}} \\
(\mathrm{min})\end{array}$ & $\begin{array}{l}\mathrm{RT}_{\text {IS }} \\
(\mathrm{min})\end{array}$ & Equation for calibration curve \\
\hline \multicolumn{4}{|c|}{ Effect of flow rate of the mobile phase $\left(\mathrm{mL} \mathrm{min}{ }^{-1}\right)$} \\
\hline 0.8 & $7.22 \pm 0.02$ & $8.52 \pm 0.02$ & $\mathrm{y}=0.0155 \mathrm{x}+0.0323\left(\mathrm{R}^{2}=0.999\right)$ \\
\hline 1.2 & $4.82 \pm 0.02$ & $5.72+0.03$ & $\mathrm{y}=0.0164 \mathrm{x}+0.0114\left(\mathrm{R}^{2}=0.997\right)$ \\
\hline \multicolumn{4}{|c|}{ Effect of mobile phase (Acetonitrile : water) } \\
\hline $10: 90$ & $6.84 \pm 0.03$ & $9.51 \pm 0.04$ & $y=0.016 x+0.026 \quad\left(R^{2}=0.995\right)$ \\
\hline $14: 86$ & $5.34 \pm 0.01$ & $5.90 \pm 0.01$ & $\mathrm{y}=0.014 \mathrm{x}+0.019 \quad\left(\mathrm{R}^{2}=0.994\right)$ \\
\hline
\end{tabular}

Table 2: Recovery, accuracy and the precision of quantification of paracetamol in human urine and plasma using HPLC/UV

\begin{tabular}{|c|c|c|c|c|c|c|c|c|}
\hline & \multirow[b]{2}{*}{$\begin{array}{c}\text { Spike } \\
\text { level } \\
\left(\mathrm{mg} \mathrm{L}^{-1}\right)\end{array}$} & \multirow[b]{2}{*}{$\begin{array}{c}\text { Recovery } \\
(\%) \\
M \pm \text { STD }\end{array}$} & \multirow{2}{*}{$\begin{array}{c}\text { Accuracy } \\
\mathrm{M} \pm \mathrm{STD} \\
\text { Observed } \\
\text { level }\left(\mathrm{mg} \mathrm{L}^{-1}\right)\end{array}$} & \multirow[b]{2}{*}{$\begin{array}{c}\text { Intra } \\
\text { day } \\
(\% \mathrm{CV})\end{array}$} & \multirow{2}{*}{$\begin{array}{l}\text { Precision } \\
\text { *Inter } \\
\text { day } \\
(\% \mathrm{CV})\end{array}$} & \multicolumn{3}{|c|}{ Freeze thaw stability } \\
\hline & & & & & & $\begin{array}{l}\text { Cycle } 1 \\
\left(\mathrm{mg} \mathrm{L}^{-1}\right) \\
24 \text { hours }\end{array}$ & $\begin{array}{c}\text { Cycle } 2 \\
\left(\mathrm{mg} \mathrm{L}^{-1}\right) \\
\text { week } 2\end{array}$ & $\begin{array}{r}\text { Cycle } 3 \\
\left(\mathrm{mg} \mathrm{L}^{-1}\right) \\
\text { week } 4\end{array}$ \\
\hline & 12.5 & $99.99 \pm 1.45$ & $11.7 \pm 0.3$ & 2.3 & 4.8 & $12.5 \pm 0.6$ & $12.6 \pm 0.2$ & $12.6 \pm 0.6$ \\
\hline Plasma & 25.0 & $100.17 \pm 0.73$ & $26.1 \pm 0.4$ & 3.0 & 2.2 & $25.5 \pm 1.2$ & $25.5 \pm 1.0$ & $26.1 \pm 0.9$ \\
\hline$(n=6)$ & 50.0 & $101.24 \pm 2.59$ & $49.6 \pm 2.0$ & 2.2 & 0.3 & $50.5 \pm 1.3$ & $49.6 \pm 1.9$ & $49.3 \pm 3.5$ \\
\hline Urine & 25.0 & $99.40 \pm 0.61$ & $25.4 \pm 0.6$ & 2.3 & 4.4 & $25.7 \pm 1.0$ & $24.8 \pm 1.2$ & $24.9 \pm 0.5$ \\
\hline \multirow[t]{2}{*}{$(n=6)$} & 50.0 & $100.81 \pm 0.50$ & $49.6 \pm 2.8$ & 3.0 & 0.8 & $50.1 \pm 1.3$ & $50.6 \pm 1.6$ & $50.8 \pm 1.9$ \\
\hline & 100.0 & $103.55 \pm 2.52$ & $101.6 \pm 2.3$ & 3.2 & 1.7 & $101.2 \pm 0.6$ & $100.6 \pm 0.6$ & $100.4 \pm 2.3$ \\
\hline
\end{tabular}

The data represent the mean $(\mathrm{M}) \pm$ standard deviation (STD) of the recovery accuracy and freeze thaw stability assays. $*_{\mathrm{n}}=10$ 
variability in measured levels over six weeks (Table 2). At $4^{\circ} \mathrm{C}$, the concentration of paracetamol was stable for at least 6 days (24 hours, fourth and sixth day) enabling the accurate measurement of unknown serum samples with the calibration curves made with repeat analysis of the same standards. The regression curve obtained using mean values ( \pm standard deviation) for gradient and intercept for post preparative stability over six days was $\mathrm{y}=0.0156( \pm 0.00) \mathrm{x}+0.010( \pm .005)$ and $\mathrm{y}=0.0167( \pm 0.00)$ $+0.018( \pm .008)$ with a regression coefficient $\left(R^{2}\right)>0.995$ for plasma and urine respectively. The calibration curves were compatible with the calibration curve obtained for the freshly made standards, indicating the stability of the processed samples. Furthermore, the concentration of paracetamol in freeze-thaw and short term stability evaluation were not significantly different from the fresh calibrators (Table 2).

The recovery of paracetamol was excellent (Table 2). Acetonitrile adequately extracted all bound paracetamol producing over 99\% recovery at all three QC levels in all matrices tested (Table 2). Similar recoveries were observed in water spiked samples suggesting minimal matrix effects on the recovery of paracetamol in plasma and urine and the values were $99.21 \pm 1.42,100.62 \pm 2.31$, $101.53 \pm 0.46$ and $100.27 \pm 0.89 \%$ for $10,25,50$ and $100 \mathrm{mgL}^{-1}$ respectively. Furthermore, matrix effects were not present in HPLC/UV analysis as the calibration slope remained unchanged despite that the standards were made in matrix or water (Figure 1).

The accuracy of the method was adequate and exceeded $94 \%$ in all matrices studied and the relative standard deviation of repeat measurements of the three QC levels ranging from 2.2-3.0 and 2.3-3.2\% were observed for plasma and urine respectively (Table 2). The interday reproducibility was presented in $\% \mathrm{CV}$ of repeated analysis of paracetamol $(n=6)$ over 45 days at the three QC levels and the values were less than 5\% for both serum and urine (Table 2). The LOD was $0.13 \mathrm{mg} \mathrm{L}^{-1}$ in plasma, and $0.68 \mathrm{mg} / \mathrm{L}$ in urine, whereas, the LOQ was $0.43 \mathrm{mg} \mathrm{L}^{-1}$ in plasma and $2.25 \mathrm{mg} \mathrm{L}^{-1}$ in urine.

In order to apply this methodology, the blood level of paracetamol in healthy volunteers in Sri Lankan population $(n=5)$ was determined at different time intervals after single administration of paracetamol at a dose of $1 \mathrm{~g}$. The serum concentration of paracetamol in blood was $10.71 \pm 3.95 \mathrm{mg} \mathrm{L}^{-1}(\mathrm{n}=5)$ at 1 hour after oral administration of paracetamol (unpublished data) at a $1 \mathrm{~g}$ dosage. The mean ( \pm standard deviation) serum paracetamol concentration of the samples sent to over laboratory for analysis in suspected paracetamol overdose was $52.06 \pm 44.77 \mathrm{mg} \mathrm{L}^{-1}(\mathrm{n}=80)$. The postmortem sample showed a paracetamol concentration of $39.7 \mathrm{mg} \mathrm{L}^{-1}$.

\section{DISCUSSION}

The preferred analytical method for urgent estimation of the plasma acetaminophen concentration is HPLC ${ }^{22}$. Most of these methods used HPLC in conjunction with solid-phase extraction with UV or electrochemical detection and sample extraction procedures that involve multiple, time-consuming organic extractions and solvent evaporation steps $^{22}$.

Positive diagnosis of paracetamol poisoning requires rapid analysis of paracetamol levels in body fluids of overdosed patients. Because of ever increasing accidental poisoning and intentional suicide attempts associated with paracetamol, a rapid analysis is essential for the effective management of paracetamol poisoning. A selective and accurate analytical method has been developed which requires minimal sample handling for quantification of paracetamol. Use of liquid-liquid extraction of paracetamol from serum was very common in most of reported methods ${ }^{23-25}$. The elimination of time consuming liquid-liquid extractions, drying and reconstitution steps in our present work minimized the processing time and the cost of sample analysis. Use of internal standard in acetonitrile provided the precipitation of protein, simultaneously minimizing the dilution of the sample compared to the other protein precipitation methods described by several authors in plasma paracetamol analysis ${ }^{26,27}$. Centrifuging at x10,000 $\mathrm{g}$ adequately removed proteins and other insoluble residues from serum, plasma and urine. No buffers were used in the present method as described in several studies ${ }^{23,24,26}$, to avoid the salt precipitation in columns and thereby extend their lifetime.

\section{Acknowledgment}

This research was sponsored by the National Research Council. The support extended by Dr Manori J. Silva, Centers for Disease Control, Atlanta, Georgia, USA for her valuable suggestions and assistance to complete this work and the technical assistance of Mr. Thisara Andrahennadi and Ms Nilusha Priyanthi, Department of Biochemistry and Molecular Biology, Faculty of Medicine, University of Colombo, are gratefully acknowledged. Volunteers involved in paracetamol pharmacokinetic studies and those who provided the samples for paracetamol analysis are also thanked. 
(A)

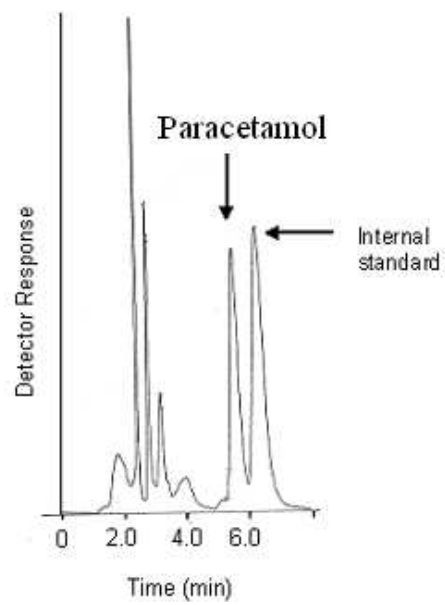

(B)

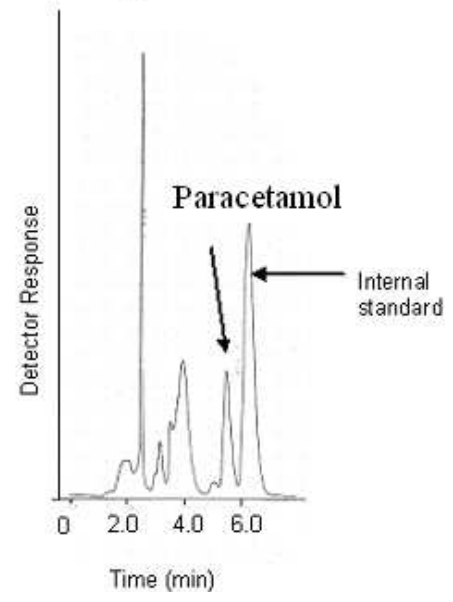

Figure 1: HPLC chromatograms of paracetamol $(5.53 \mathrm{~min})$ and the internal standard $\beta$ Hydroxyethyltheophylline (6.46 $\mathrm{min}$ ) in plasma (A) and urine (B). The peaks were identified by the retention times. The other peaks observed in the chromatograms were attributed to the substances present in matrices.
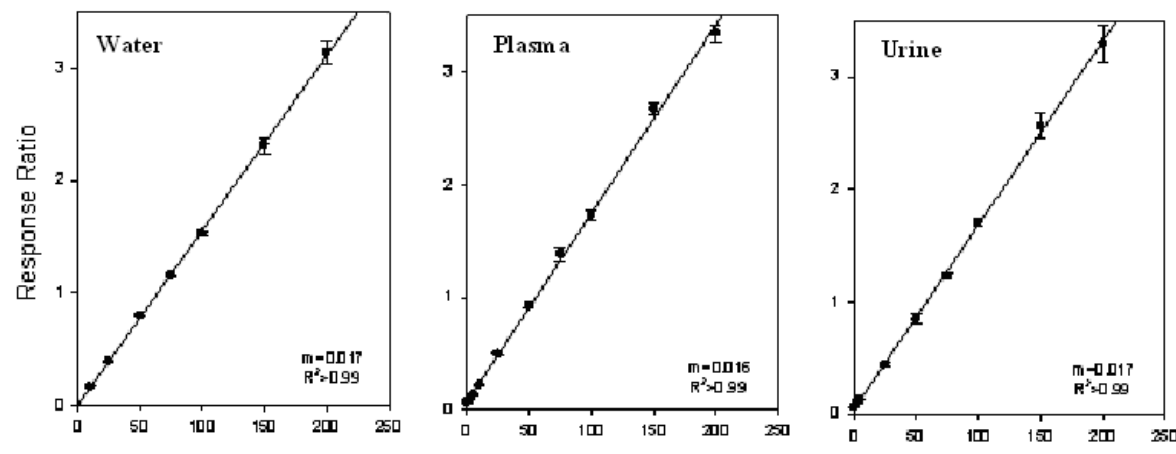

Concentration of Paracetamol, (mg $\mathrm{L}^{-b}$ )

Figure 2: Linearity of the standard curve of paracetamol in water, plasma and urine over concentration range of $0.25-200 \mathrm{mg} \mathrm{L}^{-1}$. R2 exceeds 0.99 . (Response ratio - Area under curve of paracetamol /Area under curve of internal Standard)

\section{References}

1. Moore N. (2007). Ibuprofen: a journey from prescription to over-the-counter use. Journal of the Royal Society of Medicine 100: 2-6.

2. Walsh A., Edwards H. \& Fraser J. (2007). Over-the-counter medication use for childhood fever: a cross-sectional study of Australian parents. Journal of Paediatrics and Child Health 43: 601-606.

3. Koling S., Hempel G., Lanvers C., Boos J. \& Wurthwein G. (2007). Monitoring paracetamol metabolism after single and repeated administration in pediatric patients with neoplastic diseases. International Journal of Clinical Pharmacology and Therapeutics 45: 496-503.

4. Peterson R.G. \& Rumack B.H. (1978). Toxicity of acetaminophen overdose. Annals of Emergency Medicine 7(5): 202-205.

5. Ali F.M., Boyer E.W. \& Bird S.B. (2008). Estimated risk of hepatotoxicity after an acute acetaminophen overdose in alcoholics. Alcohol 42: 213-218.
6. Black M. (1984). Acetaminophen hepatotoxicity. Annual Review of Medicine 35:577-593.

7. James L.P., Mayeux P.R. \& Hinson J.A. (2003). Acetaminophen-induced hepatotoxicity. Drug Metabolism and Disposition 31: 1499-1506.

8. Bessems J.G.M. \& Vermeulen N.P.E. (2001). Paracetamol (acetaminophen)-induced toxicity: molecular and biochemical mechanisms, analogues and protective approaches. Critical Reviews in Toxicology 31: 55-138.

9. Dahlin D.C. \& Nelson S.D. (1982). Synthesis, decomposition kinetics, and preliminary toxicological studies of pure n-acetyl-p-benzoquinone imine, a proposed toxic metabolite of acetaminophen. Journal of Medicinal Chemistry 25: 885-886.

10. Bose D., Durgbanshi A., Martinavarro-Dominguez A., Capella-Peiro M.E., Carda-Broch S., Esteve-Romero J.S. \& Gil-Agusti M.T. (2005). Rapid determination of acetaminophen in physiological fluids by liquid chromatography using SDS mobile phase and ED detection. Journal of Chromatographic Science 43: 313318. 
11. Baranowska I., Plonka J. \& Baranowski J. (2006). HPLC analysis of methylxanthines and selected drugs in urine samples. Chemia Analityczna 51: 751-760.

12. Yin O.Q.P., Lam S.S.L. \& Chow M.S.S. (2005). Simultaneous determination of paracetamol and dextropropoxyphene in human plasma by liquid chromatography/tandem mass spectrometry: application to clinical bioequivalence studies. Rapid Communications in Mass Spectrometry 19: 767-774.

13. Quattrone' A.J. \& Putnam R.S. (1981). A single liquidchromatographic procedure for therapeutic monitoring of theophylline, acetaminophen, or ethosuximide. Clinical Chemistry 27(1): 129-132.

14. Gotelli G.R., Kabra P.M. \& Marton L.J. (1977). Determination of acetaminophen and phenacetin in plasma by high-pressure liquid chromatography. Clinical Chemistry 23(6): 957-959.

15. Khatri J., Qassim S., Abed O., Abraham B., Al-Lami A. \& Masood S.A. (2001). Novel extractionless hplc fluorescence method for the determination of glyburide in the human plasma: application to a bioequivalence study. Journal of Pharmacy \& Pharmaceutical Sciences: 4(2):201-206.

16. Holland D.T., Godfredsen K.A., Page T. \& Conner J.D. (1998). Simple high-performance liquid chromatography method for the simultaneous determination of serum caffeine and paraxanthine following rapid sample preparation. Journal of Chromatography B-Analytical Technologies in the Biomedical and Life Sciences 707: 105-110.

17. Hunek C., Burgyan M. \& Wang S. (2008). Measurement of 10,11-dihydro-10-hydroxy-carbamazepine in serum and plasma by high-performance liquid chromatography. Clinical Chemistry \& Laboratory Medicine 46(10): 14291433.

18. Kulkarni R.D., Bartakke N.A., Nair A., Kadam P.P., Thatte U.M. \& Oak S.N. (2007). Pharmacokinetics of rectal compared to intramuscular paracetamol in children undergoing minor surgery. Indian Journal of Pharmacology 39: 187-191.

19. Tagliaro F., Dorizzi R., Frigerio A. \& Marigo M. (1990). Non-extraction HPLC method for simultaneous measurement of dyphylline and doxofylline in serum. Clinical Chemistry 36: 113-115.

20. Food and Drug Administration, Guidance for Industry: Bioanalytical Method Validation. http://www.fda.gov/ cder/Guidance/4252fnl.pdf. Accessed in May 2001.

21. Taylor J.K. (1987). Quality Assurance of Chemical Measurements pp. 78-83 Lewis Publishers, 121 South Main Street, Chelsea, Michign ,USA.

22. Bosch E.M., Sánchez A.J.R., Rojas F.S. \& Ojeda C.B. (2006). Determination of paracetamol: historical evolution Journal of Pharmaceutical and Biomedical Analysis 42(3): 291-321.

23. Douidar S.M. \& Ahmed A.E. (1982). Studies on simultaneous determination of acetaminophen, salicylic acid and salicyluric acid in biological fluids by high performance liquid chromatography. Journal of Clinical Chemistry \& Clinical Biochemistry 20(11): 791-798.

24. Campanero M.A., Calahorra B., Garci'a-Que'tglas E., Lo'pez-Oca'riz A. \& Honorato J. (1999). Rapid liquid chromatographic assay for the determination of acetaminophen in plasma after propacetamol administration: application to pharmacokinetic studies. Journal of Pharmaceutical and Biomedical Analysis 20: $327-334$.

25. Vertzoni M.V., Archontaki H.A. \& Galanopoulou P. (2003). Development and optimization of a reversed-phase high performance liquid chromatographic method for the determination of acetaminophen and its major metabolites in rabbit plasma and urine after a toxic dose. Journal of Pharmaceutical and Biomedical Analysis 32(3): 487-493.

26. Henderson C.J., Wolf C.R., Kitteringham N., Powell H., Otto D. \& Park K.B. (2000). Increased resistance to acetaminophen hepatotoxicity in mice lacking glutathione $S$-transferase Pi. Proceedings of the National Academy of Sciences 97(23): 12741-12745. 